\title{
Research on the Graph Matching based on Shape Context and Sequential Monte Carlo
}

\author{
Meiju Liu, Hongyu Yang, Zhaohua Chen, Lingyan Li \\ Faculty of information and control, Shenyang Jianzhu University, Shenyang, 110168, China
}

Keywords: Graph Matching; Shape Context; Sequential Monte Carlo

\begin{abstract}
According to the traditional shape context algorithm within the image distortion, excessive noise points and lower matching rate issues, we present a Sequential Monte Carlo algorithm based on graph matching. First, the image feature points were evenly distributed to compute shape context information. Second, remaining points obtain a histogram of all the feature points by the shape context information. Using histogram function to calculate the cost of the square distance cost, were begin to match. Finally, structuring the graph model, using graphical models construct the affinity matrix; the matrix will be close to integer quadratic programming use the Sequential Monte Carlo algorithm to find out the optimal matching schemes. Experimental results show that: the proposed algorithm in image matching to ensure a high rate, while images of different perspectives and images in quite different conditions has good robustness and stableness.
\end{abstract}

\section{Introduction}

Point Set Recognition as an image matching basic unit, the basic algorithm for matching have two major categories: First, based on the relationship of transformation algorithm. Second, the feature based algorithm for matching. Such issues are commonly used based on local characteristics matching scale invariant feature based on the probability of the spectrum point matching method and characteristics graph based matching. Graph theory-based approach is used for graphical models of relationship between the dot patterns, structure close to the matrix, integer quadratic programming through all points pattern matching scheme. This is the NP-Hard problems [1]. Generally has a time complexity. The traditional graph matching first used for the concept of the affinity matrix. Spectral theory first applied to the point pattern matching is Scott and LonguetHiggins [2]. But for the rotation angle of the larger set of points, the effect is not very satisfactory. To overcome the shortcomings of this method [3] Shapiro and Brady proposed a method called the affinity matrix of internal point set [4]. Carcassonne proposed a structure close to a variety of matrices; the aim is to get close to the matrix with the literature [1] of method combines. Reference [5] describes an unsupervised learning graph pattern matching algorithm. FIG proposed a deformable matching method [6], the object matching based on morphological differences, the gradient method and machine learning classification of a feature point set. [7] Proposed a tensor-based matching algorithm.

This paper proposes an algorithm of sequential Monte Carlo for graph matching. Ensure the matching accuracy, and the operational efficiency has improved. Monte Carlo method is a continuous relationship on the graph matching to find the best solutions, combined with particle filter using a graph model for processing of the best matching model. In order to measure adjust the probabilities by prior probabilities. Do not rely on the similarity of the cost function directly histogram model. An important function is to achieve through merit-based selection purposes. Given the superiority of the algorithm is proposed based on Monte Carlo cycle graph matching algorithms. The algorithm is more intuitive analysis of the optimal matching scheme, compared to the traditional graph matching algorithm greatly improves the matching result for deviations within the image distortion, light conditions. To ensure the matching accuracy and to increase the efficiency. Applicable as a result of the image is missing in some uncontrollable factors, fuzzy and matching errors caused by occlusion combine with the Shape Context.

The paper is organized as follows. In the next section, we propose the algorithms that we research in this paper, and some definitions and assumptions are given. In Section 3, the graph 
matching based on Shape Context is presented. Section 4 presents the Sequential Monte Carlo algorithm. In Section 5, the computational simulation is made to illustrate the efficiency and accuracy of the algorithm. Finally, we conclude our paper in section 6 .

\section{Discussed problems}

\section{Definition 1}

The concept of the Gradient is to find the Pixel area of rapid change. Then, the gradient is defined as follow:

$$
\begin{aligned}
& G=\sqrt{G_{x}^{2}+G_{y}^{2}} \\
& \theta=\arctan \frac{G_{y}}{G_{x}}
\end{aligned}
$$

\section{Definition 2}

The histogram of each point is to descript the characteristic of each point. The histogram can be defined as the value of this point and the remaining points is an angle.

\section{Definition 3}

For the two images, we propose the all points and graphs as well.

$$
\begin{aligned}
& P=\left\{x_{i} \mid x_{1}, x_{2}, \ldots x_{m}\right\} \\
& Q=\left\{y_{j} \mid y_{1}, y_{2}, \ldots, y_{n}\right\} \\
& G_{p}=\left(V_{p}, E_{p}\right) \\
& G_{q}=\left(V_{q}, E_{q}\right)
\end{aligned}
$$

\section{Graph Matching based on Shape Context}

Shape context is an algorithm used to describe the shape of the inner contour of the target point set rules of a descriptor. For a series of point set, shape context information describes point modes. Then, the histogram of each point and the remaining points is the angle formed by the shape of the point of the context information. To calculate the maximum similarity of histogram, the similarity cost function weights the square distances. Which the interior of the set point comprises a point radius is infinite, the focal point is the total number of points, and 0 to each area is divided into 12 sub-regions. For any points, calculate the angle remaining point (a point at each point). Finally, the histograms, Shape Context full use of context information, and reflected in the packet from non-uniform manner, thus having invariant regions. Based on the characteristics of the shape, the use of the chi-square distance histogram cost function as the similarity of shape context matching cost function:

$$
\text { Cost }=\frac{1}{2} \sum_{i=1}^{n} \frac{[g(i)-h(i)]^{2}}{g(i)+h(i)}
$$

Cost is smaller, the greater the similarity between the midpoint of the two images, the higher the probability of a match. The shape cost is as the equation (2):

$$
H(\pi)=\sum_{i} C\left(p_{i}, q_{\pi}(i)\right)
$$

According to the cost of the shape, the difference between the images can be measured.

\section{Sequential Monte Carlo algorithm}

For two images, the feature points, figure model of its points were set and posed. With the graph model, point pattern matching is transformed into graph model matching problem. Where, $\mathrm{V}$ represents a node graph model. $V(G)=\left\{v_{1}, v_{2}, \ldots, v_{n}\right\}$. The set of vertices consisting of the feature point set. And E represents a graph model consist of nodes in the undirected edges. This can be 
characterized by each connective nodes of a diagram. $v_{s}=\left(x_{i}, y_{j}\right), \quad v_{i}^{p} \in V_{p}, v_{j}^{q} \in V_{q}$. Similarity matrix $\mathrm{x}$ and $\mathrm{y}$ may be constituted by $\mathrm{X}$ can be expressed as:

$$
X=\{0,1\}^{\mathrm{n}_{\mathrm{p}} \times n_{q}}
$$

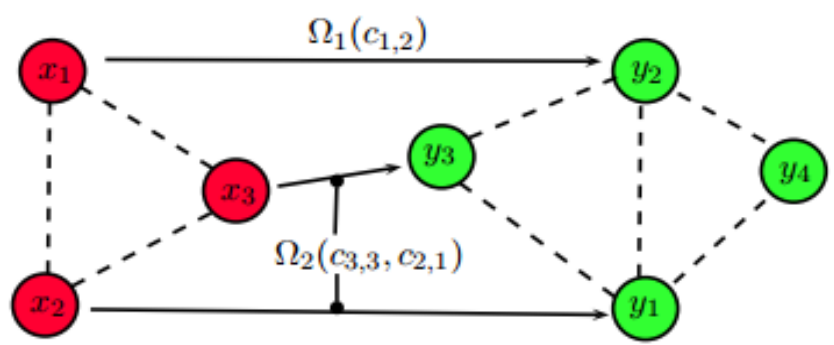

Fig.1. The Graph Matching Problem

The combination of each column of the matrix X together can form a column vector. This can construct the affinity matrix $\mathrm{K}$. The elements of $\mathrm{K}$ can be expressed as:

$$
k_{i_{1} i_{2} j_{1} j_{2}}=\left\{\begin{array}{l}
k_{i_{1} i_{2}}^{p} ; i_{1}=j_{1}, i_{2}=j_{2} \\
k_{c_{1} c_{2}}^{q} ; i_{1} \neq j_{1}, i_{2} \neq j_{2} \\
0 ; \text { otherwise }
\end{array}\right.
$$

The elements of the affinity matrix in K diagram shows the matching model points and edges. According to all its matching programs, equation (5) can be derived as optimal solution.

$$
x^{*}=\arg \max \left(x^{T} K x\right) \quad \text { s.t. } \quad x \in\{0,1\}^{n_{p} \times n_{q}}
$$

This is an IQP problem.

For all integer quadratic programming solution that can match the target graph model as a sample in the probability density at time t can be represented by a series of random samples. We present the importance of the probability density of the sample. The status of each sample and the state transition equation related to satisfying the first Markov process. That is, only with relevant. By the state transition equation, the importance of the probability density distribution function can be expressed as:

$$
\eta_{t}\left(x_{t}\right)=\sum_{x_{t-1}} \pi_{t-1}\left(x_{t-1}\right) \kappa\left(x_{t-1}, x_{t}\right)
$$

Status weights at each time can be expressed as:

$$
W_{t}^{(i)}=\frac{\pi_{t}\left(x_{t}^{(i)}\right)}{\eta_{t}\left(x_{t}^{(i)}\right)}
$$

The normalized weights:

$$
\omega_{t}^{(i)}=\frac{W_{t}^{(i)}}{\sum_{i=1}^{N} W_{t}^{(i)}}
$$

As a measure weight of the samples, when the total number of samples tends to infinity, converges to its estimated value. Namely:

$$
\tilde{\pi}_{t}\left(x_{t}\right)=\sum_{i=1}^{N} \omega_{t}^{(i)} \delta_{x_{t}^{(i)}}\left(x_{t}\right)
$$

For a certain measure of constant, continuous allocation target graph model to meet:

$$
\pi_{t}\left(x_{t}\right) \propto \exp \left(x_{t}^{T} K x_{t} / \tau\right)
$$

Particle updating state restrictions as follows:

$$
m_{w}=\arg \min e_{m}^{T} K \tilde{X}_{t}
$$




\section{Computational examples and analysis}

For different image matching algorithm SM, RRWM, PM, GAGM centralized algorithm and experimental comparison. In the simulation experiment, for the images in the database matching the time and matching accuracy of comparison, the radius of the shape of set of points contained within the context of the set of points is set to infinity. And select its state transition equation initial value 0 . The following Figs and Tables show the matching rates and time efficiency of our algorithm and other algorithms. Compare with each other.

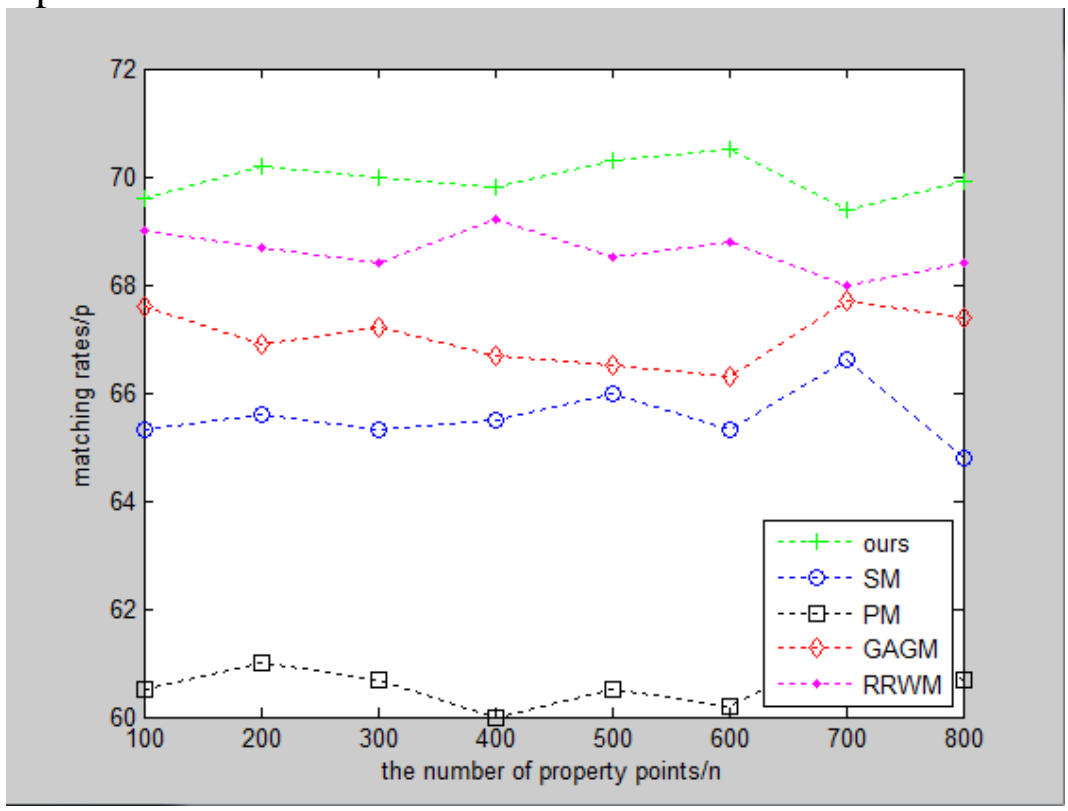

Fig.2. The matching rates of proposed algorithms

The following table shows the matching rates of all proposed algorithms.

Table1: The matching rates data

\begin{tabular}{|l|l|l|l|l|l|}
\hline \multirow{2}{*}{$\begin{array}{l}\text { The use of } \\
\text { points }\end{array}$} & \multicolumn{5}{|c|}{ Matching rates\% } \\
\cline { 2 - 6 } & ours & SM & PM & GAGM & RRWM \\
\hline P 1 & 69.6 & 65.3 & 60.5 & 67.6 & 69.0 \\
\hline P 2 & 70.2 & 65.6 & 61.0 & 66.9 & 68.7 \\
\hline P 3 & 70.0 & 65.3 & 60.7 & 67.2 & 68.4 \\
\hline P 4 & 69.8 & 65.5 & 60.0 & 66.7 & 69.2 \\
\hline P 5 & 70.3 & 66.0 & 60.5 & 66.5 & 68.5 \\
\hline P 6 & 70.5 & 65.3 & 60.2 & 66.3 & 68.8 \\
\hline P 7 & 69.4 & 66.6 & 61.3 & 67.7 & 68.0 \\
\hline P 8 & 69.9 & 64.8 & 60.7 & 67.4 & 68.4 \\
\hline
\end{tabular}




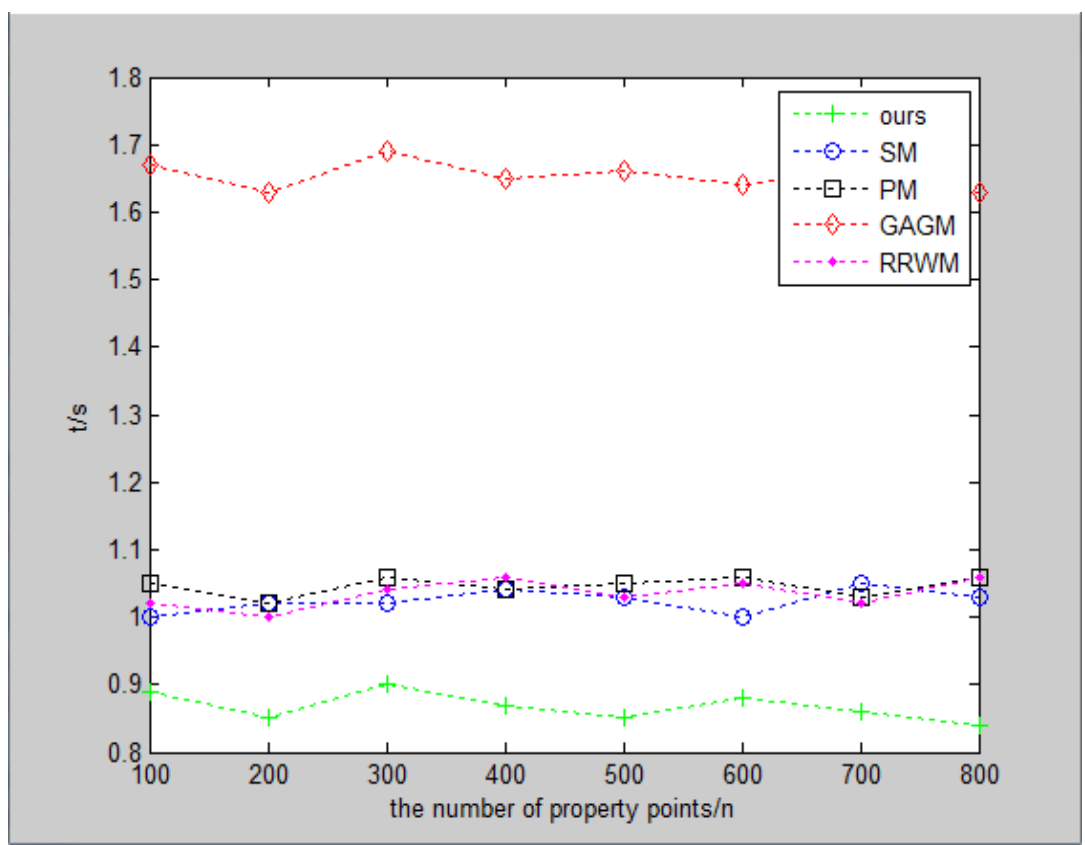

Fig.3. The time efficiency of proposed algorithms

The following table shows the time efficiency of all proposed algorithms.

Table2 :The time efficiency data

\begin{tabular}{|l|l|l|l|l|l|}
\hline algorithms & ours & SM & PM & GAGM & RRWM \\
\hline time(s) & 0.89 & 1.00 & 1.05 & 1.67 & 1.02 \\
\hline
\end{tabular}

By the matching results, matching algorithm accuracy is higher than other algorithms listed in timeliness is also better than other algorithms edge.

\section{Conclusions}

The proposed map matching algorithm based on sequential Monte Carlo, the first set of points using shape contexts extracted histogram using the chi-square distance early match, full use of context information for different sets of feature points to build graphical models, combined with particle filter effectively match the model according to FIG. Ensure precise matching rate, while also improved timeliness. Compared to similar algorithm has some advantages. This algorithm while maintaining high matching shape context, enhanced matching accuracy and computational efficiency, so this algorithm in image distortion and large differences still has good robustness. In this paper, algorithms and other similar algorithms are also compared the experimental data and experimental results show that for the original algorithm and the proposed algorithm compared to effectively save time and eliminate the ambiguity

points.

In this paper, our combined algorithm has certain advantages compared. The advantage of this method is improved with a simple structure, and the calculation amount is relatively small.

\section{Acknowledgements}

In this paper, the research was sponsored by the project of Department of Liaoning Province (Project No. L2013225)

\section{References}

[1] Duchenne, O., Bach, F., Kweon, I., \& Ponce, J. (2009). A tensor-based algorithm for high-order graph matching. InInternational Con-ference on Computer Vision and Pattern Recognition. 
[2] Ren, X. (2007). Learning and matching line aspects for articulated ob-jects. In International Conference on Computer Vision and Pat-tern Recognition.Schellewald, C., \& Schnorr, C.

[3] O. Duchenne, A. Joulin, and J. Ponce. A graph-matching kernel for object categorization. In ICCV, 2011. 1

[4] A. Myronenko and X. B. Song. Point set registration: Co-herent point drift. IEEE Trans. Pattern Anal. Mach. Intell.,32(12):2262-2275, 2010. 7

[5] Cho, M., Lee, K.M.: Progressive graph matching: Making a move of graphs via probabilistic voting. In: CVPR (2012)

[6] oggia, P., Percannella, G., Vento, M.: Graph matching and learning in pattern recognition in the last 10 years. IJPRAI (2014)

[7] Kim, V.G., Li, W., Mitra, N.J., DiVerdi, S., Funkhouser, T.: Exploring collectionsof 3D models using fuzzy correspondences. In: SIGGRAPH (2012)

[8] Tian, Y., Yan, J., Zhang, H., Zhang, Y., Yang, X., Zha, H.: On the convergence of graph matching: Graduated assignment revisited. In: Fitzgibbon, A., Lazebnik, S., Perona, P., Sato, Y., Schmid, C. (eds.) ECCV 2012, Part III. LNCS, vol. 7574, pp. 821-835. Springer, Heidelberg (2012)

[9] Gold, S., Rangarajan, A.: A graduated assignment algorithm for graph matching. IEEE Transaction on PAMI (1996)

[10] Pachauri, D., Kondor, R., Vikas, S.: Solving the multi-way matching problem by permutation synchronization. In: NIPS (2013)

[11] T. Cour, P. Srinivasan, and J. Shi. Balanced graph matching. In B. Sch“ olkopf, J. Platt, and T. Hoffman, editors, Advances in Neural Information Processing Systems 19, pages 313-320. MIT Press, Cam-bridge, MA, 2007.

[12] H. F. Wang and E. R. Hancock. Correspondence matching using kernel principal components analysis and label consistency constraints. Pattern Recognition, 39(6):1012 - 1025, 2006.

[13] B. Luo and E. Hancock. Structural graph matching using the em algorithm and singular value decomposition. IEEE Transactions on Pattern Analysis and Machine Intelligence, 23:1120-1136, 2001.

[14] A. Raj and R. Zabih. A graph cut algorithm for generalized image de-convolution. In ICCV '05: Proceedings of the Tenth IEEE International Conference on Computer Vision, pages 1048-1054, Washington, DC,USA, 2005. IEEE Computer Society.

[15] A. C. Berg, T. L. Berg, and J. Malik. Shape matching and object recognition using low distortion correspondences. In Proceedings, IEEE Conference on Computer Vision and Pattern Recognition, volume 1, pages 26-33, Washington, DC, USA, June 2005. IEEE Computer Society. [16] T. Cour, P. Srinivasan, and J. Shi. Balanced graph matching. In B. Sch“ olkopf, J. Platt, and T. Hoffman, editors, Advances in Neural Information Processing Systems 19, pages 313-320. MIT Press, Cam-bridge, MA, 2007. 